\title{
A Mandami Fuzzy Controller for Handling a OpenHAB Smart Home
}

\author{
Pablo PICO-VALENCIA ${ }^{\mathrm{a}}$ and Juan A. HOLGADO-TERRIZA ${ }^{\mathrm{b}, 1}$ \\ ${ }^{a}$ Pontificia Universidad Católica del Ecuador, Esmeraldas, Ecuador \\ ${ }^{\mathrm{b}}$ Universidad de Granada, Granada, Spain
}

\begin{abstract}
Fuzzy control systems are widely used to carry out control actions where variables with a certain degree of ambiguity are involved. Such control actions are modelled based on fuzzy rules that are expressed in a language analogous to that used by humans to conceive their reality. In this sense, fuzzy controllers can be applied in emerging technologies such as the Internet of Things (IoT) aimed at controlling the real world through a set of objects interconnected to the Internet. This article presents a fuzzy controller of Mamdani-type developed in Matlab. This controller is integrated into a smart home-created by the OpenHAB tool- using RESTful services. The results of the evaluation reveal that the integration of the fuzzy controller with smart home can provide an accurate control of comfort specifically in this case. In addition, a better organization of the control rules was also possible using the proposed fuzzy controller especially when large systems are developed.
\end{abstract}

Keywords. fuzzy controller, Mamdani, internet of things, smart home, OpenHAB

\section{Introduction}

Fuzzy logic is an alternative to the classic logic that proposes the modelling of systems where variables are handled with a certain level of imprecision (i.e., temperature, air pollution and tank filling level). These kind of variables can take ambiguous values such as: very-low, low, medium, high, very-high [1]; empty, low, medium, full, critical [2], among others. Handling this type of variables could not be supported by a precise mathematical model to establish control actions [3]. However, the application of fuzzy logic based on fuzzy sets and rules for the creation of systems enables the execution of control actions using ambiguous variables more accurately.

A fuzzy logic based control system consists of three elements: a fuzzifier, an inference engine and a defuzzifier [4]. The fuzzifier allows the variables to be modelled by means of fuzzy sets. The elements of each involved fuzzy set have an associated membership degree and they use a certain membership function according to the nature of the problem (e.g., triangular, trapezoidal, Gaussian). After the fuzzification of input and output variables of the control system, the control rules are determined. These rules of type IF-THEN are used by the inference engine to perform fuzzy inference obtaining the value of the output variable(s). Finally, the output variable(s) are defuzzified to become

\footnotetext{
${ }^{1}$ Corresponding Author: Software Engineering Department, University of Granada, E.T.S. de Ingenierías Informática y de Telecomunicación, 18071 Granada, Spain; E-mail: jholgado@ugr.es.
} 
real values from which control actions are performed on the system. To carry out this process, defuzzification methods such as centroid, bisector, min and max are used [5].

Recently, fuzzy control systems have been integrated into emerging paradigms such as the Internet of Things (IoT). In IoT ecosystems, the real world is controlled and tracked by monitoring the data provided by objects or things that are interconnected to the Internet [6]. However, it is also required to carry out control actions on the environment where humans daily live in order to satisfy their needs. In this sense, fuzzy controllers have been introduced into the IoT to control scenarios where imprecise data have to be sensed. Some of these systems are oriented for controlling automatic irrigation systems [7], lighting in smart homes [8], optimization the conditions of cultivation in greenhouses [9], controlling of pollution indoor [10], among others. These systems comply with the specifications of the most widespread fuzzy models, Mamdani or Takagi-Sugeno [11].

Since fuzzy controllers have been integrated into the IoT to control objects and communications, it is necessary to work on fuzzy controllers compatible with connected digital homes (smart homes) - scenarios where flexible and accurate control is required to satisfy the user needs. This has been one of the main motivations for this study whose objective is to integrate a Matlab fuzzy controller into a smart home built with a standard home gateway such as OpenHab [12]. Based on this integration via Restful web services, an evaluation of the controller was proposed, determining the accuracy to control smart home objects as well as the flexibility to create the control strategy; that is, defining how the set of rules was organized from which the IoT ecosystem is driven.

This paper is organized as follows. Section 2 describes how fuzzy controllers have been applied on connected digital homes based on the IoT. Section 3 describes the architecture of system as well as the process followed to integrate the fuzzy controller with the IoT. The results of the evaluation of the fuzzy controller are discussed in the section 4. Finally, conclusions and future work are outlined in section 5.

\section{Literature Review}

The main proposed fuzzy controllers for controlling household based on IoT scenarios showed that fuzzy controllers were developed in order to manage certain aspects related to comfort indoor, that is, lighting, thermal, and security. To do that, heterogeneous sensors of different providers (i.e., temperature, humidity, $\mathrm{pH}$ sensors) were installed at both indoor and outdoor of smart homes. Fuzzy controllers managed the data acquired by these sensors as main data input to perform the fuzzyfication, fuzzy inference and defuzzyfication tasks. Next, a description on the six recovered proposals after performing the scientific search are described in deep.

In most cases, the Mamdani-type fuzzy controller was used as the primary fuzzy control model [11]. In the management of thermal comfort, a first study, proposed in [13], presented a solution capable of optimizing the indoor thermal comfort condition according to seasonality (summer condition). The system developed by the researchers included IoT objects such as temperature, wind speed, fan, humidity sensors. From these devices it was possible to capture the contextual information on a smart home. Such information allowed the authors, using a linear regression model, to predict the Predictive Mean Vote (PMV) Index - a model that stands among the most disseminated models for evaluating indoor thermal comfort. Fuzzy controller used this index to compute the 
control signal by applying the predefined control rules. The model was developed in Java with the jFuzzyLogic library. The system was tested with an HVAC in a real building located in Jeju, Korea in 2017.

In the same line of thermal comfort, additional studies were proposed. In [14], a fuzzy system for controlling indoor temperature via a Mamdani model using the type2 fuzzy logic was proposed. The developed system, programmed in IT2F environment, was oriented to manage the most appropriate indoor temperature with respect to outdoor ambient temperature, maintaining at the same time a comfortable climate for users. Among the modelled fuzzy variables, there were used the following: outdoor temperature and humidity. Managing the temperature of a specific room considering the outdoor conditions, a model was enabled to control the indoor temperature not only on humidity and temperature but also on the number of people in the room. This model is ideal for smart cities because is callable of saving energy and money.

In order to achieve the goals of saving energy and money, in [15] was proposed a similar controller capable to save around $40 \%$ of energy. Although authors in this study managed the same variables for the controller, they worked with different hardware and software technologies. For instances, researchers used the ThinkSpeak as an open IoT platform for interconnecting objects, Arduino UNO and Raspberry Pi as microcontrollers for connecting sensors, and jFuzzyLogic for modelling the fuzzy system.

Likewise, Mamdani type fuzzy controllers have also been applied to light comfort control in addition to temperature management for smart homes. Accordingly, a first study, proposed in [16], developed a fuzzy luminance control system loaded in an ESP 8266 microcontroller programmed in C language. They defined two input variables (error and error change between luminance set value and measured value) and one output variable (PWM duty cycle increment value). From these variables the system could effectively maintain a stable luminance level in an office environment as a part of a smart home. Thus, the system contributes positively to power saving efficiency.

Similarly to [16] but oriented to external systems such as intelligent cities, in [17], a Mamdani fuzzy system was proposed for controlling the intensity of street lights. This system developed in Matlab could adjust the streetlights intensity level based on the temporal and environmental conditions, that is, clear sky, cloudy, foggy and traffic density. Although the proposed system was adapted to the conditions of Islamabad in Pakistan, it could be adapted for operating according the same conditions to other cities of the world.

Finally, it was also highlighted two proposals focused on aspects related to security management applicable to IoT. A first study, proposed in [18], presented a framework called Fuzzy approach to the Trust Based Access Control (FTBAC) that can be used to calculate fuzzy trust values for any number of devices which makes it more suitable for scalable loT. To do it, the fuzzy controller employed three variables that describe devices, that is, values of experience, knowledge and recommendation. This is a general framework that could be applied in devices of smart homes, but it has not been experimented.

Complementary, in [19], a trust aware access control system using fuzzy logic is described to ensure that only trusted users/devices access the data of sensor devices such as successful forward ratio, energy consumption rate and data integrity. From these data, the fuzzy controller was able to assist in the decision making of access control as well as calculate the context dependent trustworthiness of each device based on their previous behaviour. Therefore, this is a good alternative in smart homes because security and privacy are yet concerns in IoT. 


\section{Proposal}

\subsection{Scenario of IoT}

The objective of the IoT scenario was controlling the room lighting level of a smart home. In this sense, the illumination indoor of the three rooms (bathroom, master room, living room) must be automatically controlled according the activity done in the room and the contextual conditions where the smart home is located. In order to develop this IoT ecosystem (Figure 1) it was used the OpenHAB platform [12] as smart home gateway. This platform provides a vendor and technology agnostic open-source automation software for integrating heterogeneous IoT devices available in a smart home. Thus, Open$\mathrm{HAB}$ was selected because it serves as an integration way for all home automation needs and lets systems talk to each other across any vendor or protocol.
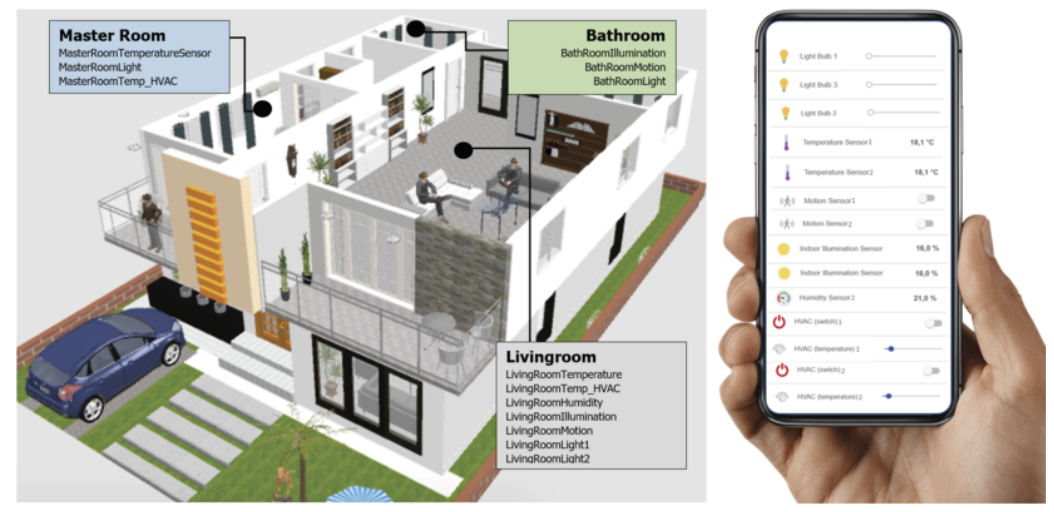

Figure 1. Scenario of IoT developed using OpenHAB.

The main IoT objects that were part of the described scenario, shown in Figure 1, included the following IoT objects: four light bulbs, two motion sensors and two illumination sensors. There were other objects as part of the scenarios but they were not used for lighting comfort. Files that implements the sitemap, items and rules of the studied scenario in OpenHAB are accessible in the project repository.

Each IoT object had associated a state and some data obtained from the linked sensor or actuator. For accessing to the status of the sensors, a Matlab Restful web service linked to the IoT sensor or actuator was invoked using the request-response model of the Hypertext Transfer Protocol Secure (HTTPS). A GET method was applied to obtain the status and data from sensors as data input of fuzzy controller. On the other hand, a PUT method was called to update the status of actuators, once the values are computed through the fuzzy controller. Both methods were accessed using uniform resource identifier (URIs) and a HTTPS client.

\subsection{Fuzzy Controller Architecture}

For the development of the proposed fuzzy controller in this study, the specifications of the general fuzzy controller model described in $[4,9]$ were followed. This means that the 


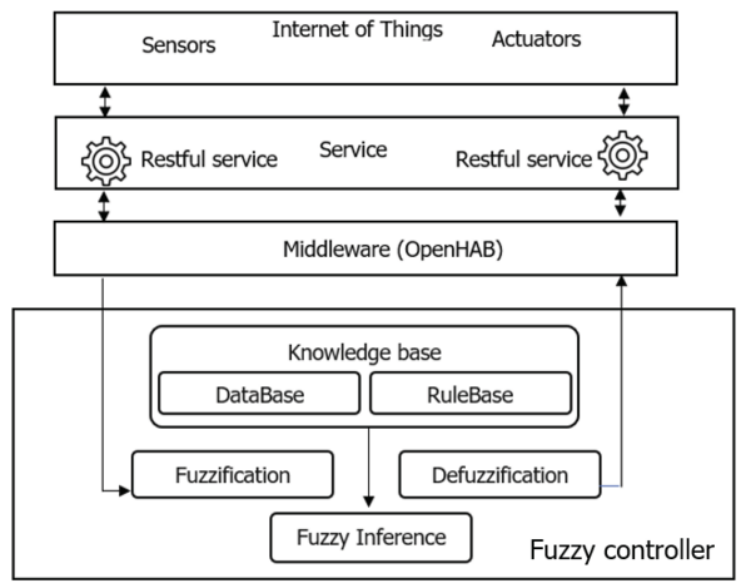

Figure 2. General architecture of the proposed fuzzy controller.

designed controller integrated the four basic components such as: a fuzzifier, a knowledge base, an inference engine and a defuzzifier. These ones are shown in Figure 2.

The schema of a fuzzy controller shown in Figure 2 defines a generic fuzzy controller that gets a set of inputs represented by fuzzy sets through the fuzzifier from the sensors of IoT ecosystem. From these variables, the rules were defined and based on them, the value of an output variable was inferred. This output variable was converted to a real value through the defuzzifier to determine the control actions to be applied to actuators. After defining the scheme of the fuzzy controllers to be developed, we determined the fuzzy logic library available for programming the fuzzy system in Matlab R2018b, that is, selecting the methods of the toolbox for fuzzy inference system (FIS).

\subsection{Design of the Fuzzy Controller}

To meet the control goals of the system (providing lighting comfort) one fuzzy controller was designed. This controller called OpenHABF uzzyLigthingComfort was oriented to control the lighting in the smart home illustrated in Figure 2, that is, in the bathroom and living room in our case. The motion and illumination sensors were used as input data to model the control actions. The fuzzy sets of the variables illumination and motion, fuzzified from both triangular and trapezoidal functions, are illustrated in Table 3.

After the fuzzyfication of the input and output variables of the proposed Mamdani controller, the fuzzy rules of the fuzzy controller were then formulated. For the controller OpenHABF uzzyLightingComfort 12 rules were defined. The antecedents (IF) and the consequent of the rules (THEN) are detailed in Table 1. It is important to emphasize that in both cases the AND operator was used to join more than two antecedents of the rules.

Finally, the centroid method was selected for the defuzzyfication of the output variable, because it is one of the most used to perform fuzzy control providing a more accurate value in relation to the other existing ones. 


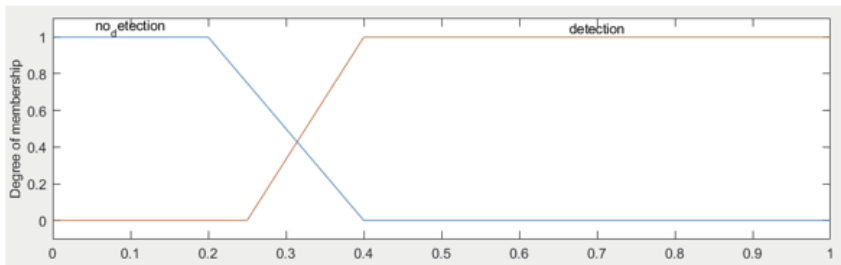

(a) Motion

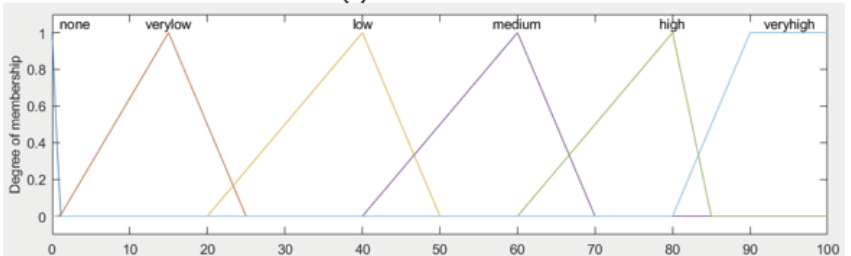

(b) Illumination

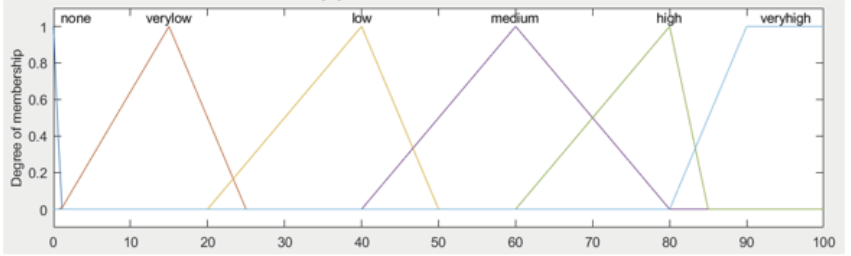

(c) LightInsentity

Figure 3. Fuzzy input and output variables for lighting control.

Table 1. Fuzzy rules for lighting control in the studied scenario.

\begin{tabular}{lll|l}
\hline Rule & Illumination $\left(i_{1}\right)$ & Motion $\left(i_{2}\right)$ & Lights $\left(o_{1}\right)$ \\
\hline 1 & none & no_detection & none \\
2 & very_low & no_detection & none \\
3 & low & no_detection & none \\
4 & medium & no_detection & none \\
5 & high & no_detection & none \\
6 & very_high & no_detection & none \\
7 & none & detection & very_high \\
8 & very_low & detection & high \\
9 & low & detection & medium \\
10 & medium & detection & low \\
11 & high & detection & none \\
12 & very_high & detection & none \\
\hline
\end{tabular}

\subsection{Development of the Fuzzy Controller}

The controllers designed were developed using the library for fuzzy systems provided by Matlab R2018b. In general, the process for creating the fuzzy controller for handling lighting comfort (OpenHABLightingComfort) consisted in the creation of a fuzzy control, fuzzyfication of the two input variables such as motion and illumination, fuzzyfication of the output variable and finally, the definition of the rules that drive the controller as well as the setting of these rules to the previously fuzzy control created.

Once the fuzzy controller was created, it was executed continuously applying a control cycle on the studied modelled smart home. Listing 1 describes the implementation of 


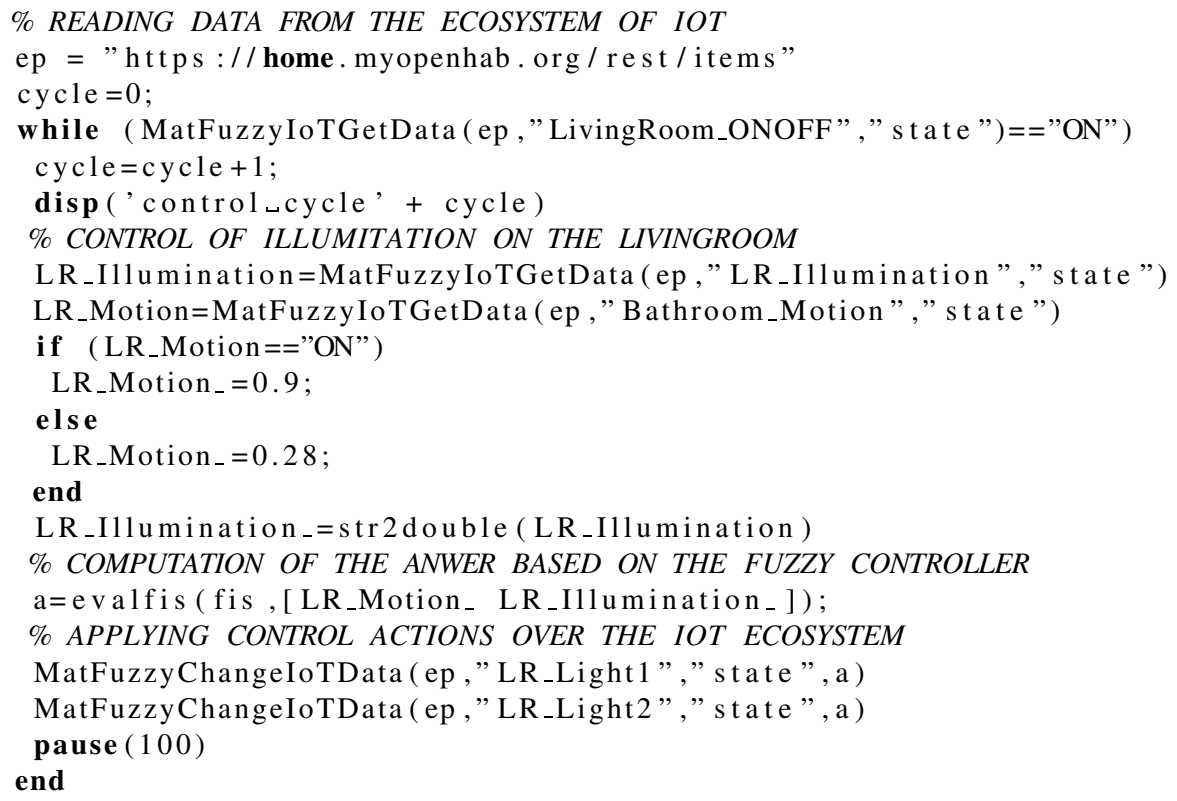

Listing 1. Continuous cycle for controlling thermal comfort over the studied scenario of IoT.

illumination control for the living room called OpenHABLightingComfortRun. In line 4 , the endpoint of deployed RESTful service is defined for reading input data from the IoT ecosystem such as illumination and motion (lines 10-17). Then, those data were used to compute the output variable using the fuzzy controller OpenHABLightingComfort, that is lightbulb intensity (line 19). Finally, the obtained value was used to change the state of the lightbulb of the IoT ecosystem (lines 21-23). In a similar way, the lighting was controlled on the bathroom. This script was executed while the operation switch installed on the smart home is in state "ON".

\section{Results and Discussion}

\subsection{Evaluation of the Accuracy of Output}

An evaluation of the system was carried out to validate its effectiveness in the control actions applied to the ecosystem of IoT under study. The results for all cases were coherent with the designed fuzzy controller. This implies the level of illumination in the studied scenario was as expected. Some particular examples changing data in the GUI of the IoT ecosystem, such as motion (M) and illumination (I) for controlling the lightbulb intensity (L) are described as follows:

The discourse universe of illumination and lightbulb intensity had a range from 0 to 100 . For lightbulb intensity, we connected 4000 kelvin Hue light bulbs with 806 lumens while for illumination a Xiaomi smart light sensor was selected with a variability 
Table 2. Results of the evaluation of the proposed fuzzy controller on the IoT ecosystem.

\begin{tabular}{ll|l}
\hline Illumination $\left(i_{1}\right)$ & Motion $\left(i_{2}\right)$ & Lights $\left(o_{1}\right)$ \\
\hline 0 & 0.28 & 0 \\
0 & 0.8 & 92.5 \\
12 & 0.8 & 74.8 \\
38 & 0.8 & 60 \\
55 & 0.8 & 13.6 \\
71 & 0.8 & 0 \\
94 & 0.8 & 0 \\
\hline
\end{tabular}

from 0 to 83000lux. The motion was detected by using a Hue motion sensor. Based on these ranges handled by the fuzzy controller, the values calculated by the defuzzyfication method allowed the application of automatic control processes adapted to reality and to the needs required in the rooms under study. However, the applied control is static because only a specific user pattern was considered. Therefore, it is recommended to include within the controller other variables related to user needs, that is, modes of use, user preferences, among others. This will enable the system adaptation to many more cases and to different user needs.

It is known that regarding fuzzy controllers, many works published in the literature have used Matlab's fuzzy logic library to develop controllers similar to the one proposed in this work. Its wide use has been one of the motivations for using this tool. The results obtained in this study allowed to determine the effectiveness of the controller in the IoT developed using OpenHAB. The level of intensity of the lightbulb managed from OpenHAB was carried out consistently and accurately according to the rules defined.

\subsection{Evaluation of Response Time}

An evaluation of the response time of the system was also carried out to execute the control cycle to manage light and thermal comfort in the studied IoT ecosystem. This evaluation was made on a Lenovo branded personal computer with a $2.50 \mathrm{GHz}$ i7 processor and 16 GB of RAM. The computer is installed with the 64-bit Windows 8.1 operating system and the Java 1.8 virtual machine. Furthermore, the ecosystem of IoT, implemented with OpenHAB 2.0 ran on an HP personal computer with $1.6 \mathrm{GHz}$ i5 processor and $8 \mathrm{~GB}$ of RAM. This ecosystem can be accessed via Internet because the cloud connector of OpenHAB was used.

In Figure 4, it can be observed that the response time required to run the light control system, which controlled the lightbulbs located in the bathroom and the living room, was $14.188 \mathrm{~s}$. This result was obtained after calculating the mean after running the control loop OpenHABLigthingComfortRun for 10 iterations. The dispersion of the response times has a standard deviation of $0.902 \mathrm{~s}$. All values were accessed over the cloud of OpenHAB using the HTTPS protocol over the Internet. The connection speed was 50 Mbps.

In summary, the developed system was executed efficiently. For a scenario such as an smart home that does not need hard real time, the times obtained are acceptable. However, such time may change when the system is running in an environment where IoT devices must be accessed on an intranet instead of the Internet. It is recommended that only those specific IoT objects that should be accessed from anywhere outside the au- 


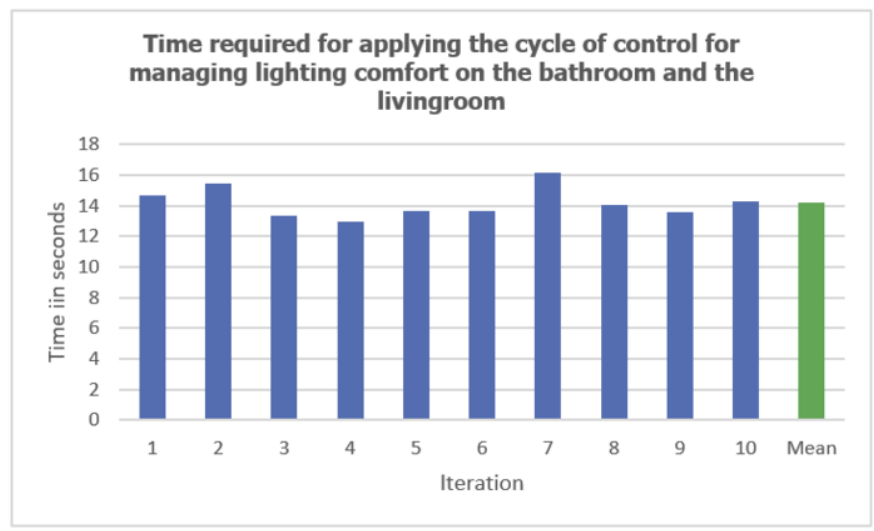

Figure 4. Time required for executing cycle of continuous control of lighting comfort.

tomated scenario may use the OpenHAB cloud connector in order to gain access to it. The remaining objects can be consumed by fuzzy controller via the intranet. These recommendations will considerably increase the overall efficiency of the developed system and can be applied to time-constrained tasks if needed.

\section{Conclusions and Future Work}

IoT ecosystems require intelligent mechanisms to carry out communications and control actions of the interconnected actuators and sensors as accurately as possible. To develop these mechanisms, Artificial Intelligence plays an important role. Techniques such as fuzzy control systems have helped designing and creating intelligent systems. In this sense, fuzzy logic is relevant because it allows dealing with variables that have some ambiguity. In the case of smart homes, the use of environmental conditions (i.e., temperature, humidity, lighting) supports some needs regarding the management of thermal, lighting and safety comfort to make its users feel more comfortable.

The use of IoT platforms, with services support, for the management of resources and communications between devices, contributes significantly to the integration of fuzzy controllers with IoT networks. Its use not only helps to capture data from the environment required by the controllers to carry out changes on actuators, but also allows the use of cloud computing to run the inference implemented by fuzzy controllers. This allows fuzzy controllers to be used in resource-constrained environments. As the results show, the application of fuzzy control does not require sophisticated resources. Furthermore, the possibility of applying control in undefined environments is not synonymous of decreasing in the effectiveness of control, which is generally done with simple rules.

As future work, we propose the development of an add-on oriented to the development of fuzzy controllers compatible with IoT in a way that abstracts the complexity of smart home systems when they are integrated in IoT networks as well as it facilitates the creation of additional smart scenarios such as hospitals, industry, city, smart university, among others. It also proposes the use of fuzzy variables to model not only conditions directly related to intelligent systems (i.e., temperature), but also user preferences (modes of use, preferences). This would facilitate the management of preferences in areas where 
there are multiple users, an aspect that is generally complex to model in order to satisfy more than single users.

\section{References}

[1] Ameen NM, Al-Ameri JAM. IoT-Based Shutter Movement Simulation for Smart Greenhouse Using Fuzzy-Logic Control. In: 2019 12th International Conference on Developments in eSystems Engineering (DeSE). IEEE; 2019. p. 635-39.

[2] Anduray RGJ, Irigoyen SMZ. Development of a fuzzy controller for liquid level by using raspberry pi and internet of things. In: 2017 IEEE Central America and Panama Student Conference (CONESCAPAN). IEEE; 2017. p. 1-5.

[3] Alcala-Fdez J, Alonso J. A Survey of Fuzzy Systems Software: Taxonomy. Current Research Trends and Prospects. 2015;24(1):40-56.

[4] Petritoli E, Leccese F, Cagnetti M. Takagi-Sugeno Discrete Fuzzy Modeling: an IoT Controlled ABS for UAV. In: 2019 II Workshop on Metrology for Industry 4.0 and IoT (MetroInd4. 0\&IoT). IEEE; 2019. p. 191-95.

[5] Talon A, Curt C. Selection of appropriate defuzzification methods: Application to the assessment of dam performance. Expert Systems with Applications. 2017;70:160-74.

[6] Ng ICL, Wakenshaw SYL. The Internet-of-Things: Review and research directions. International Journal of Research in Marketing. 2017;34(1):3-21.

[7] Alomar B, Alazzam A. A Smart Irrigation System Using IoT and Fuzzy Logic Controller. In: 2018 Fifth HCT Information Technology Trends (ITT). IEEE; 2018. p. 175-79.

[8] Lu Y, Wang J, Bai X, Wang H. Design and implementation of LED lighting intelligent control system for expressway tunnel entrance based on Internet of things and fuzzy control. International Journal of Distributed Sensor Networks. 2020;16(5).

[9] Carrasquilla-Batista A, Chacón-Rodríguez A. Standalone Fuzzy Logic Controller Applied to Greenhouse Horticulture Using Internet of Things. In: 2019 7th International Engineering, Sciences and Technology Conference (IESTEC). IEEE; 2019. p. 574-79.

[10] Pradityo F, Surantha N. Indoor Air Quality Monitoring and Controlling System based on IoT and Fuzzy Logic. In: 2019 7th International Conference on Information and Communication Technology (ICoICT). IEEE; 2019. p. 1-6.

[11] Nguyen AT, Taniguchi T, Eciolaza L, Campos V, Palhares R, Sugeno M. Fuzzy control systems: Past, present and future. IEEE Computational Intelligence Magazine. 2019;14(1):56-68.

[12] Heimgaertner F, Hettich S, Kohlbacher O, Menth M. Scaling home automation to public buildings: A distributed multiuser setup for OpenHAB 2. In: 2017 Global Internet of Things Summit (GIoTS). IEEE; 2017. p. 1-6.

[13] Hang L, Kim DH. Enhanced Model-Based Predictive Control System Based on Fuzzy Logic for Maintaining Thermal Comfort in IoT Smart Space. Applied Sciences. 2018;8(7):1031.

[14] Jana DK, Basu S. Novel Internet of Things (IoT) for controlling indoor temperature via Gaussian type-2 fuzzy logic. International Journal of Modelling and Simulation. 2019;41(2):1-9.

[15] Meana-Llorián ea. IoFClime: The fuzzy logic and the Internet of Things to control indoor temperature regarding the outdoor ambient conditions. Future Generation Computer Systems. 2017;76:275-84.

[16] Xiaole Y, Chunlai Y, Zhe Y, Hongbo Y, Gang P. Power Saving LED Luminance Fuzzy Control with IOT Network. In: IOP Conference Series: Materials Science and Engineering. vol. 435. IOP Publishing; 2018. p. 012059.

[17] Sultan K. Fuzzy Rule Based System (FRBS) assisted Energy Efficient Controller for Smart Streetlights: An approach towards Internet-of-Things (IoT). Journal of Communications. 2018;13(9):518-23.

[18] Mahalle PN, Thakre PA, Prasad NR, Prasad R. A fuzzy approach to trust based access control in internet of things. In: Wireless VITAE 2013. IEEE; 2013. p. 1-5.

[19] Thirukkumaran R, Muthukannan P. TAACS-FL: trust aware access control system using fuzzy logic for internet of things. International Journal of Internet Technology and Secured Transactions. 2019;9(12):201-20. 